\title{
Philosophical investigations into the essence of pediatric suffering
}

\author{
Tyler Tate T,2 $^{1,2}$
}

Accepted: 27 October 2020 / Published online: 17 December 2020

(c) Springer Nature B.V. 2020

Baby Esther (or Essie, as her parents have taken to calling her) is a prematurely born eight-day-old neonate with severe lissencephaly syndrome who is currently residing in the neonatal intensive care unit. Lissencephaly-a rare genetic brain malformation characterized by the absence of normal folds in the cerebral cortex and an abnormally small head-was a devastating diagnosis for Esther's parents, who had been trying to get pregnant for over seven years. Symptoms of lissencephaly syndrome include profound intellectual disability, unusual facial appearance, difficulty swallowing, inability to grow, muscle spasms, seizures, severe motor impairment, and deformities of the hands, fingers, and toes. Children with severe lissencephaly require total and continuous care in order to survive and rarely live past the age of ten. On the morning of Esther's ninth day of life, her medical team discovers that she needs surgery to remove a section of necrotic intestine if she is to have any chance of survival. Esther's parents-who have a strong Christian faith-and her clinicians are both conflicted about the operation. Each wonders if Esther's constrained future and vast potential for suffering recommend against surgery. A nurse raises the question of what can be known, if anything, about the nature and extent of Esther's suffering, as it exists either now or in the future.

$* * *$

Childhood suffering is one of life's most disturbing realities. Although all suffering is prima facie bad, the suffering of children is uniquely tragic. To most people living in the contemporary West, childhood suffering seems particularly gruesome and deeply unfair: children, with all their cherubic innocence, do not deserve to suffer or to die premature deaths. ${ }^{1}$ This attitude both grounds the logic of pediatric

\footnotetext{
${ }^{1}$ Historian Joseph Amato observes how "we read in the paper of a child who was beaten by his own parents until his buttocks were turned to a jelly-like pulp. Or we read of a child torn to bits by two pit bulls. Unless we lack sensitivity altogether, we are compelled, at least on some interior level, to react to this suffering - to its waste, stupidity, unfairness, and cruelty. There seems to us something so unfair, so

Tyler Tate

tatety@ohsu.edu

1 Department of Pediatrics, Oregon Health and Science University, Portland, OR, USA

2 Center for Ethics in Health Care, Oregon Health and Science University, Portland, OR, USA
} 
medicine, with its fastidious emphasis on curative therapies and the eradication of genetic disease, and provides a starting point for health care systems to fundraise for child health, build freestanding children's hospitals, and grow pediatric research programs.

Yet when probed further, the conceptual stability and sociopolitical implications of pediatric suffering become convoluted. Suffering, which is difficult enough to define in adults who can communicate when they suffer, is all the more vague when applied to children. As seen in the story of baby Esther, a turbid cloud of suffering often hangs over severely ill children. Put less obliquely, clinicians and parents frequently discuss childhood suffering while simultaneously feeling uncertain about whether, when, how, and to what extent a patient suffers - and if a patient is deemed to be suffering, they question what medical decisions that suffering can justify. The concept of pediatric suffering becomes increasingly nebulous when considering children who are newly born or have significant cognitive impairments. Patients within these categories, who cannot speak for themselves, depend completely on others to both describe their suffering and craft treatment plans that will attempt to alleviate it.

One way to bring the role that suffering plays in the practice of pediatric medicine and ethics into sharper focus is to examine it alongside the related concept "quality of life" [2]. In pediatrics, lives with quality and lives worth living go hand in hand. In other words, if a life has quality, it is considered a life worth living [3, pp. 136-142]. However, to actually shape the practice of pediatrics, what constitutes "quality" must be made explicit. Defining and filling out notions of pediatric quality of life depend deeply on conceptions of suffering. For example, in his book Death or Disability?, an extended analysis of decision-making for impaired neonates, Dominic Wilkinson identifies a level of impairment and degree of projected poor quality of life that together make a neonate's life not worth living. If an impaired neonate's condition is predicted to "impose substantial burdens on the child" and "render it highly likely that the infant or child will have a life not worth living and be significantly harmed by continuing treatment," then the continuation of treatment is not ethically permissible [4, p. 277]. Wilkinson recognizes the indeterminacy of many of these terms, and much of the book is dedicated to their clarification. Yet when he finally puts his framework to use on a patient—a hypothetical child with severe brain injury, ongoing seizures, and permanent ventilator dependence-suffering is his final recourse. He concludes that for such children, "very dramatic reduction in benefits from life combined with evidence that they may be suffering makes it very likely that continuing life-sustaining treatment would harm them" [4, p. 294]. For Wilkinson, then, it is suffering that ultimately justifies death over disability.

More recently, Erica Salter has explored the influence of suffering on the shaping of ethical judgment in pediatric practice. In her essay "The new futility? The rhetoric and role of 'suffering' in pediatric decision-making," Salter notices how claims of child suffering can consciously or unconsciously "smuggle value judgments" into ethical deliberations that might otherwise be regarded as even-handed

Footnote 1 (continued)

outrageous, about the manifestly purposeless suffering of these children.... We rebel against the idea of purposeless suffering. It seems unjust and intolerable” [2, pp. xvii-xviii]. 
and value-neutral [5, p. 17]. She argues that suffering has become the new qualitative "futility" for children [6], with descriptions of suffering used to mask the inherent subjectivity of clinical appraisals of quality of life for pediatric patients with disabilities and to motivate and justify the removal of their life-sustaining treatments. Her concern is not an isolated one. Other writers, focusing mainly on the Netherlands' Groningen Protocol for neonatal euthanasia, have also contended that claims of suffering can conceal biases regarding what kinds of lives are worth living [7, 8]. Salter supports her arguments with a qualitative content analysis that she conducted alongside Annie Friedrich and Kirsten Dempsey [9]. Their findings reveal that out of 651 instances of the term "suffering" in 121 pediatric ethics articles published in 2007-2017, 52\% are used to support a specific medical decision. Moreover, suffering is three times more likely to be invoked in support of a life-ending decision (32\%) than in support of a life-extending decision (10\%).

As a pediatric palliative care doctor, I share Salter's concerns. I work in a profession where witnessing and attempting to alleviate suffering are built into the job description. In this job, I have found time and time again how easy it is for clinicians assessing and theorizing about childhood suffering to rely on patterns of reasoning dictated by raw emotional responses or tacit cultural conditionings. This malleability is disconcerting given the tremendous influence the concept of suffering holds within the practice and discourses of pediatric medicine, as the above examples from the pediatric ethics literature illustrate. Suffering has traction in people's lives: when a child is said to be suffering, action follows. Unfortunately, the existing literature on childhood suffering is marked by under-theorization and an overall lack of precision with regard to metaphysics (what is suffering, especially for someone who cannot speak or participate in discursive reasoning?) epistemology (how can one know if a child is suffering or will suffer?), and ethics (how should one respond to the suffering of someone who cannot communicate?).

The aim of this Theoretical Medicine and Bioethics special issue is to critically engage the complex metaphysical, epistemological, and ethical issues surrounding childhood suffering. The goal of this engagement is conceptual and moral clarity. Yet, recalling the words of Wendell Berry's small-town barber in the novel Jayber Crow, no act of description is complete: "Telling a story is like reaching into a granary full of wheat and drawing out a handful. There is always more to tell than can be told" [10, p. 29]. Philosophical analysis is as diverse as the human beings who perform it, and there is always more to be said. A sense of restive incompleteness is evident in all of the essays in this special issue. This, I believe, is one of the collection's distinct strengths. In engaging the case of baby Esther, the essays in this special issue approach the philosophical problem of pediatric suffering from a set of unique perspectives that reflect the authors' diverse philosophical traditions, disciplinary formations, and clinical experiences. Suffering has many faces.

In the first article, I argue that pediatric suffering must be understood not as a mental state, but as a judgment or evaluation [11]. I start by analyzing the different ways that the label of suffering is used in pediatric practice, distilling what I call the twin poles of pediatric suffering. One pole is characterized by the belief that infants like Esther cannot suffer because they do not have language and subjective life experience. The other pole holds the idea that once child suffering reaches a 
certain threshold, it is better to eliminate the sufferer than to allow the suffering to continue unabated. At both poles, the particular child at hand drops out of sight, leaving behind a bare simulacrum. In an attempt to locate a theory that accommodates the experiences of children, I examine Jamie Mayerfeld's and Michael Brady's so-called experiential accounts of suffering $[12,13]$, finding them both to be inadequate on the basis of their absurd entailments and their flawed assumptions regarding the subjective experiences of people without language, such as infants and those with severe neurocognitive impairment. Instead, drawing on the work of Alasdair MacIntyre [14], I argue that child suffering is best understood as a set of absencesabsences of conditions that would otherwise constitute child flourishing. Such an understanding of suffering brings the profound dependency of children into focus while also helping to elucidate the inherently social and political nature of pediatric suffering. As opposed to adults, children are not responsible for their own suffering, nor can they allay it. Rather, children rely wholly on others to resist suffering, grow, and flourish.

In the second essay, Gina Campelia, Jennifer Kett, and Aaron Wightman offer a reorientation of the metaethical framework typically used in clinical ethics to evaluate pediatric suffering [15]. The authors do not believe that the nexus of baby Esther's suffering can be identified with something intrinsic to her, such as her pain or her distress, thus circumventing the thorny epistemic problem of infant suffering. Rather, they argue that the claim by parents or clinicians that baby Esther is suffering grows out of the loving interpersonal relationships that Esther exists within. It is not baby Esther alone, but Esther-in-relation that screams of pain and loss. In other words, for Campelia, Kett, and Wightman, baby Esther's suffering is not equivalent to her pain, seeing that one can never fully know if she is feeling pain or even having subjective experience. In addition, contra what Eric Cassell believes, her suffering does not lie in a lack or loss of meaning, purpose, or agency (cf. [16]). Rather, Campelia and colleagues contend, it is in their relationships that humans find and feel significant emotional and moral distress and thereby proceed to identify the presence of suffering. In this way, a closer look at the meshwork of relationships surrounding a child like Esther can provide a more robust understanding of whether and why suffering is present in any case at hand. This novel method of inquiry draws attention to the relations in which baby Esther is defined and through which she may flourish or suffer. Such an epistemic reorientation allows the authors to sketch a constructive account of how to respond to baby Esther's suffering, a response they provocatively call the "moral authority of love and care" [15].

For Dominic Wilkinson and Amir Zayegh, the central question for Esther's parents and the clinicians caring for her is whether having her life prolonged by surgery is in Esther's best interests [17]. To answer this question, the authors seek to analyze two further questions: (1) What is the value or disvalue of a very short life? (2) How does profound cognitive impairment impact on suffering and quality of life? In response to the first question, they posit that because a fetus cannot experience pleasure or distress, fetal life has no prudential, or objective, value. In this sense, a fetus is like gold or the tallest redwood in California: it cannot have rights, and it obtains "value" only contingently by being valued. Extending this position to a newborn, Wilkinson and Zayegh argue that the principal value of infant life lies ahead of the infant-it is constituted both in the infant's inchoate subjective experience and 
in the realization of future objectively valuable elements of well-being. Although Wilkinson and Zayegh remain agnostic about the overall prudential value of life for an infant with cognitive impairment who dies at six weeks of age, like baby Esther, they ultimately contend that her value is at best mildly positive. Therefore, surgery may be recommended. They contrast this view with that of David Benatar, who has argued that all very short lives are intrinsically bad because distress will always outweigh whatever meager pleasures a newborn can experience [18].

To analyze the second question about the impact of profound cognitive impairment on suffering and quality of life, Wilkinson and Zayegh explore a volume control analogy, according to which global neurological impairment may attenuate both positive and negative subjective experiences, just as reducing the volume on a stereo reduces both pleasant and unpleasant sounds. For them, the ratio of attenuation between positive and negative subjective experience is key. They see three options: (a) positive and negative subjective experiences reduce symmetrically; (b) the experience of subjective ills reduces more dramatically, engendering a "tolerability paradox" [19] whereby a neurologically impaired infant may actually be better off than a healthy infant; or (c) the experience of pleasures decreases more quickly than the experience of pains, in what they call the dyshedonia effect, such that the lives of some infants like Esther are not worth living - in other words, Esther would be better off dead. Although Wilkinson and Zayegh remain ambivalent about which choice is correct, their intuitions seem to point to the third option: they do not think babies with profound neurological impairment should be kept alive indefinitely if there is no hope for improvement and the content of their subjective experience if overwhelmingly negative. A subsequent analysis of the capacity of medicine to relieve Esther's suffering further supports their intuitions; they argue that it is possible that Esther will experience unrelieved suffering in spite of palliative medicine's best efforts. Taken all together, then, for Wilkinson and Zayegh, the burden of proof is on those who do not affirm the dyshedonia hypothesis.

Finally, John Lantos offers a commentary on the preceding three papers and presents his own analysis of the case of baby Esther [20]. To that end, Lantos explores the complex psychology of Bird, the semi-autobiographical character from Kenzaburo Oe's novel A Personal Matter [21]. Bird becomes the father of an infant with a serious brain anomaly and, like Esther's parents, he must wrestle with questions about the baby's suffering and what that suffering would compel him to do for his child. This metaphor of wrestling is apt: as Lantos ultimately sees it, like wrestling, the process of describing suffering and determining how to respond to it takes work, and that work cannot be done alone. It is only in deliberative communities - whether construed broadly as the field of bioethics or more narrowly as a particular intensive care unit - that the moral life can be articulated, and a way through the quagmire of pediatric suffering can be charted.

As a bit of editorial housekeeping, the idea for these papers germinated in a panel discussion at the 2019 Conference on Medicine and Religion in Durham, North Carolina, and then gathered more steam and coherence at the 2019 Pediatric Bioethics Conference in Seattle, Washington. During the publication process, my paper, Campelia, Kett, and Wightman's paper, and Zayegh and Wilkinson's paper were each subjected to anonymous peer review by two or three separate content experts. I prepared and managed this review process, and my brother, David Tate, reviewed 
and helped me to edit both this introduction and John Lantos's paper. Together, these five papers make up this Theoretical Medicine and Bioethics special issue on pediatric suffering. My hope is that they will enlighten readers and inspire further critical engagement with this important topic.

\section{References}

1. Amato, Joseph. 1990. Victims and values: A history and theory of suffering. New York: Praeger Publishers.

2. Desai, Arti D., Chuan Zhou, Susan Stanford, Wren Haaland, James W. Varni, and Rita M. Mangione-Smith. 2014. Validity and responsiveness of the pediatric quality of life inventory (PedsQL) 4.0 generic core scales in the pediatric inpatient setting. JAMA Pediatrics 168: 1114-1121.

3. Ross, Lainie Friedman. 1998. Children, families, and health care decision-making. New York: Oxford University Press.

4. Wilkinson, Dominic. 2013. Death or disability? The "Carmentis Machine" and decision-making for critically ill children. Oxford: Oxford University Press.

5. Salter, Erica K. 2020. The new futility? The rhetoric and role of "suffering" in pediatric decisionmaking. Nursing Ethics 27: 16-27.

6. Schneiderman, Lawrence J., Nancy S. Jecker, and Albert R. Jonsen. 1990. Medical futility: Its meaning and ethical implications. Annals of Internal Medicine 112: 949-954.

7. Kodish, Eric. 2008. Paediatric ethics: A repudiation of the Groningen protocol. Lancet 371: 892-893.

8. Kon, Alexander A. 2008. We cannot accurately predict the extent of an infant's future suffering: The Groningen protocol is too dangerous to support. American Journal of Bioethics 8(11): 27-29.

9. Friedrich, Annie B., Kirsten M. Dempsey, and Erica K. Salter. 2020. The use of suffering in pediatric bioethics and clinical literature: A qualitative content analysis. Pediatric Ethicscope 27(1): $16-27$.

10. Berry, Wendell. 2000. Jayber Crow. Washington, DC: Counterpoint.

11. Tate, Tyler. 2020. What we talk about when we talk about pediatric suffering. Theoretical Medicine and Bioethics 41. https://doi.org/10.1007/s11017-020-09535-8.

12. Mayerfeld, Jamie. 1999. Suffering and moral responsibility. New York: Oxford University Press.

13. Brady, Michael. 2018. Suffering and virtue. Oxford: Oxford University Press.

14. MacIntyre, Alasdair. 1999. Dependent rational animals: Why human beings need the virtues. Chicago: Open Court.

15. Campelia, Georgina D., Jennifer C. Kett, and Aaron Wightman. 2020. Relational suffering and the moral authority of love and care. Theoretical Medicine and Bioethics 41. https://doi.org/10.1007/ s11017-020-09530-z.

16. Cassell, Eric J. 2004. The nature of suffering and the goals of medicine, vol. 2. New York: Oxford University Press.

17. Wilkinson, Dominic, and Amir Zayegh. 2020. Valuing life and evaluating suffering in infants with life-limiting illness. Theoretical Medicine and Bioethics 41. https://doi.org/10.1007/s11017-02009532-x.

18. Benatar, David. 2006. Better never to have been: The harm of coming into existence. Oxford: Clarendon Press.

19. Wilkinson, D. 2006. Is it in the best interests of an intellectually disabled infant to die? Journal of Medical Ethics 32: 454-459.

20. Lantos, John. 2020. Our suffering and the suffering of our time. Theoretical Medicine and Bioethics 41. https://doi.org/10.1007/s11017-020-09529-6.

21. Oe, Kenzaburo. 1968. A personal matter. Trans. John Nathan. New York: Grove Press.

Publisher's Note Springer Nature remains neutral with regard to jurisdictional claims in published maps and institutional affiliations. 